Organic vapor discrimination with chemiresistor arrays of temperature modulated tin-oxide nanowires and thiolate-monolayer-protected gold nanoparticles

This article has been downloaded from IOPscience. Please scroll down to see the full text article.

2011 Nanotechnology 22125501

(http://iopscience.iop.org/0957-4484/22/12/125501)

View the table of contents for this issue, or go to the journal homepage for more

Download details:

IP Address: 141.211.173.82

The article was downloaded on 06/04/2012 at 16:54

Please note that terms and conditions apply. 


\title{
Organic vapor discrimination with chemiresistor arrays of temperature modulated tin-oxide nanowires and thiolate-monolayer-protected gold nanoparticles
}

\author{
K Scholten ${ }^{1,2}$, F I Bohrer ${ }^{1,3}$, E Dattoli ${ }^{1,4}$, W Lu ${ }^{1,4}$ and \\ E T Zellers ${ }^{1,3,5,6}$ \\ ${ }^{1}$ Center for Wireless Integrated Microsystems, University of Michigan, Ann Arbor, \\ MI 48109-2122, USA \\ 2 Applied Physics Program, University of Michigan, Ann Arbor, MI 4819-1040, USA \\ ${ }^{3}$ Department of Chemistry, University of Michigan, Ann Arbor, MI 4819-1055, USA \\ ${ }^{4}$ Department of Electrical Engineering and Computer Science, University of Michigan, \\ Ann Arbor, MI 4819-2122, USA \\ ${ }^{5}$ Department of Environmental Health Sciences, University of Michigan, Ann Arbor, \\ MI 4819-2029, USA \\ E-mail: ezellers@umich.edu
}

Received 13 November 2010, in final form 15 November 2010

Published 14 February 2011

Online at stacks.iop.org/Nano/22/125501

\begin{abstract}
This paper explores the discrimination of organic vapors with arrays of chemiresistors (CRs) employing interface layers of tin-oxide nanowires (NWs) and thiolate-monolayer-protected gold nanoparticles (MPNs). The former devices use contact-printed mats of NWs on micro-hotplate membranes to bridge a pair of metal electrodes. Oxidation at the NW surface causes changes in charge transport, the temperature dependence of which differs among different vapors, permitting vapor discrimination. The latter devices use solvent cast films of MPNs on interdigital electrodes operated at room temperature. Sorption into the organic monolayers causes changes in film tunneling resistance that differ among different vapors and MPN structures, permitting vapor discrimination. Here, we compare the performance and assess the 'complementarity' of these two types of sensors. Calibrated responses from an NW CR operated at two different temperatures and from a set of four different MPN CRs were generated for three test vapors: n-hexane, toluene, and nitromethane. This pooled data set was then analyzed using principal components regression classification models with varying degrees of random error superimposed on the responses via Monte Carlo simulation in order to estimate the rates of recognition/discrimination for arrays comprising different combinations of sensors. Results indicate that the diversity of most of the dual MPN-CR arrays exceeds that of the dual NW-CR array. Additionally, in assessing all possible arrays of 4-6 CR sensors, the recognition rates of the hybrid arrays (i.e. MPN $+\mathrm{NW}$ ) were no better than that of the 4-sensor array containing only MPN CRs.
\end{abstract}

\section{Introduction}

Heated films of tin-oxide have been used in chemiresistors (CRs) for gases and vapors for over 30 years; resistance

6 Author to whom any correspondence should be addressed. changes accompanying analyte oxidation at the surface of the tin-oxide permit highly sensitive detection of a wide range of analytes [1]. Nanowires (NWs) of tin-oxide have been reported to offer potentially improved sensitivity to gases and vapors by virtue of their high ratio of surface area to volume and improved degree of crystalline order [2-5]. 
(a)

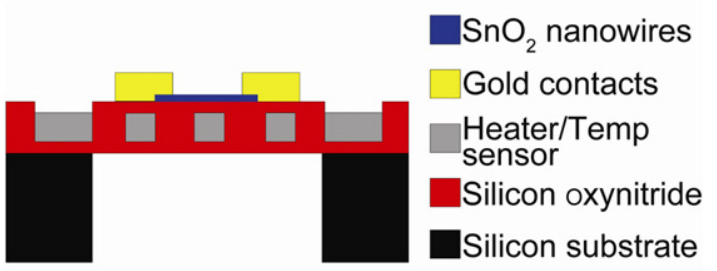

(b)

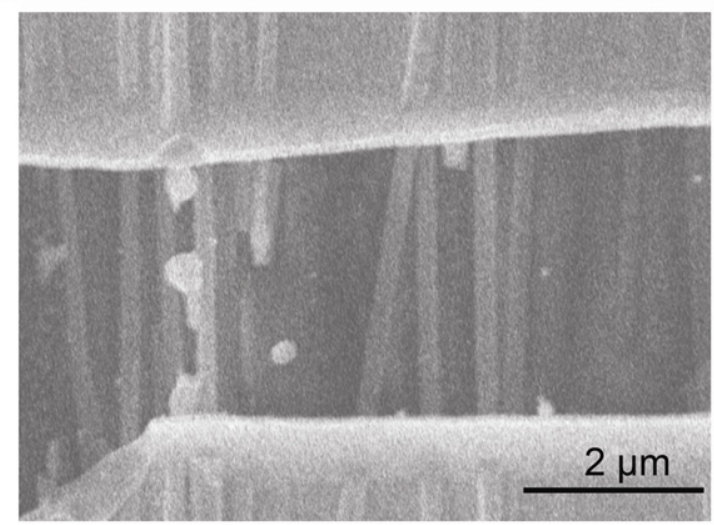

(c)

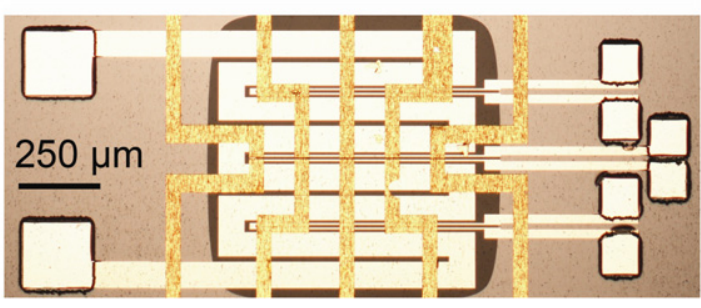

Figure 1. (a) Cross sectional diagram of the NW CR on the membrane hotplate; (b) scanning electron microscope (SEM) image of the tin-oxide NW mat bridging the CR electrodes;

(c) photomicrograph of the central region of the NW-CR chip with five electrode pairs distributed horizontally across the membrane hot plate.

(This figure is in colour only in the electronic version)

Deposition of NWs onto micromachined hotplate platforms can yield low-power temperature-modulated NW-CR sensors with responses to different analytes that exhibit different temperature dependences $[3,6]$. Although recent studies suggest that it should be possible to discriminate among a set of organic vapors by means of an array of as few as two such devices operated at different (elevated) temperatures [3, 4, 6-8], this topic has not been addressed in published reports.

Thiolate-monolayer-protected Au nanoparticles (MPNs) have been studied as interface materials for CR vapor sensors over the past 12 years [9-15]. Vapor sorption into the organothiolate monolayers surrounding the Au cores in a thin MPN film increases the average intercore distance, which reduces the rate of electron tunneling and generally increases the film resistance. If the dielectric constant of the vapor is significantly greater than that of the intercore matrix, this factor will mitigate swelling-induced resistance changes [16, 17]. An array of CRs with different MPN interface layers will exhibit partially selective responses whose pattern can be used to discriminate among individual vapors and the components of simple mixtures. Several reports have shown this capability using arrays of 2-4 sensors [11, 16, 18, 19].

Since the mechanisms by which resistance changes arise in these two classes of sensors are completely different, it might be expected that an array of NW CRs would provide relative responses to a set of analyte vapors that would complement those provided by an array of MPN CRs. Thus, greater diversity and selectivity might be realized from a hybrid $[\mathrm{NW}+\mathrm{MPN}]-\mathrm{CR}$ array. The notion of combining sensors operating on the basis of different transduction or interaction mechanisms is not new [20-24], and recent studies of multitransducer arrays of capacitive, calorimetric, and gravimetric sensors employing polymer interface layers suggest that some improvement in the diversity of responses to a set of vapors and their mixtures can be realized, relative to single-transducer arrays containing the same number of sensors [25, 26]. As yet, however, no study has explored whether combining NW CRs with MPN CRs in hybrid arrays might yield improvements in vapor discrimination over that obtained with CR arrays employing one or the other type of interface material. The primary goal of the preliminary investigation described here was to test this hypothesis.

Toward that end, data were collected from a set of CRs coated with four different MPNs operated at ambient temperature and also from an NW CR operated at two different (elevated) temperatures upon exposure to each of three individual vapors. The calibrated responses were then pooled and the various actual and virtual arrays of 2-6 sensors were evaluated with regard to vapor discrimination. Monte Carlo simulations were used to iteratively generate synthetic responses by superimposing different levels of random error on the calibrated sensor responses, and then principal components regression analysis was used to estimate the rates of recognition (RR) achievable among the different possible arrays. Assessments were made of the diversity of responses exhibited using each type of interface material (i.e. NWs and MPNs) as well as the degree to which they complement each other in effecting vapor discrimination. For this study, tests were confined to differentiation among three individual vapors (i.e. no analyses of mixtures were performed).

\section{Materials and methods}

The NW CR is a multi-layer structure in which a silicon oxynitride membrane supports a resistive heater electrically insulated from the top NW layer by another oxynitride layer (figure 1(a)). First, the Ti-Pt resistive heater and temperature sensor were defined by a standard liftoff process on a $1 \mu \mathrm{m}$ silicon oxynitride insulating layer pre-deposited on a $\mathrm{Si}$ substrate. A second $1 \mu \mathrm{m}$ layer of silicon oxynitride was then deposited over the Ti-Pt structures, with contact pads subsequently exposed by an HF etch. Next, NWs, fabricated as described previously [27], were transferred to the device surface as a mat of randomly oriented nanowires 
via contact printing [28]. Five pairs of Au contacts, $50 \mu \mathrm{m}$ wide and separated by 2-4 $\mu \mathrm{m}$, were lithographically defined and deposited on top of the NWs by a second liftoff process (figure 1(b)). Finally, the backside of the Si substrate was subjected to deep reactive ion etching to yield a $2 \mu \mathrm{m}$ thick suspended membrane structure. Figure 1(c) shows the central region of a completed chip, with the five electrode pairs aligned horizontally over the membrane region.

Electrical connections were made by wire bonds and each device was mounted on a 20-pin ceramic header. Although devices could be heated to $>360^{\circ} \mathrm{C}$, initial testing resulted in several device failures from stress-induced cracking of the membrane substrate upon cooling. The likely cause of this problem is the mismatch in thermal expansion of the heater metals and the underlying/overlying substrate layers arising from the fact that the metals were deposited at room temperature and not annealed. Subsequent tests were constrained to temperatures $\leqslant 360{ }^{\circ} \mathrm{C}$. It required $275 \mathrm{~mW}$ to heat the devices to $300{ }^{\circ} \mathrm{C}$. Resistance was measured with a multimeter (34401A, Agilent Technologies, Palo Alto, CA) and, by convention, converted to conductance. Sensor responses are reported as the fractional change in conductance relative to the baseline value (i.e. $\Delta G / G$ or $G_{\mathrm{r}}$ ). Only one device per chip was used for testing.

An array of eight MPN CRs was created as described previously [29]. $\mathrm{Cr}-\mathrm{Au}$ interdigital electrodes with $5 \mu \mathrm{m}-$ wide fingers and spaces were deposited on a thick $\mathrm{SiO}_{2}$ layer grown on a $\mathrm{Si}$ substrate. Pins were soldered to the bonding pads and plugged into sockets on a custom PC board that connected the devices to a voltage divider circuit. Interface layers were made by solvent casting films of MPNs having thiolate monolayers derived from n-octanethiol (C8), 4-(phenylethynyl)-benzenethiol (DPA), 6-phenoxyhexane-1thiol (OPH), and methyl-6-mercaptohexanoate (HME) [30]. Two films of each type of MPN were deposited. The resistance was determined indirectly by measuring the voltage drop using a computer controlled DAQ card and a constant DC voltage source. By convention, sensor responses are reported as the fractional change in resistance (i.e. $\Delta R / R$ or $R_{\mathrm{r}}$ ).

A single mounted NW CR was sealed in a 0.51 glass chamber equipped with ports for electrical feedthroughs and gas flow. The MPN-CR array was sealed in a 0.51 stainless steel chamber immediately upstream from the NW$\mathrm{CR}$ chamber. The flow rate of the test-atmosphere through the chambers was $141 \mathrm{~min}^{-1}$. Nitromethane, n-hexane, and toluene were the analytes tested. Test atmospheres were generated by passing scrubbed air through fritted bubblers containing the (single) liquid analyte, followed by dilution with a metered scrubbed air stream. The flows were varied to span a range of concentrations, which differed for each analyte, but was within the range of 200-7000 ppm (by volume). Vapor concentrations were confirmed by a calibrated flame ionization detector. Typical tests entailed exposure for 57 min followed by purging with clean air for a similar amount of time. Responses to n-hexane and toluene were collected simultaneously from the MPN-CR array and the NW CR over a three-four-fold concentration range. Responses to nitromethane were collected in separate, sequential exposures to each type of sensor at two discrete concentrations for the NW CRs and over a six-fold range for the MPN-CR array. The net change between the (average) pre-exposure baseline sensor signal under scrubbed air and the steady-state signal observed during vapor exposure was recorded.

The performance of the various actual and virtual arrays was assessed using Monte Carlo simulations coupled with extended disjoint principal components regression (EDPCR) classification models. Using the experimental sensitivity values, synthetic MPN-CR responses to each vapor were generated by randomly selecting a vapor concentration within the range of 3-30 $\times$ LOD, where the LOD was dictated by the least sensitive sensor in the array to ensure that all sensors contributed to the response patterns. The response was calculated from the calibration-curve regression equation. Then, error was introduced by adding a value obtained by multiplying that response value by a factor derived from randomly sampling a Gaussian distribution with a mean of zero and a standard deviation of $0.05,0.10$, or 0.15 , corresponding to random sensitivity errors $(\varepsilon)$ of $5-15 \%$ of the response. The error enhanced responses from all sensors were combined and the location of the resulting response vector was projected onto the principal component corresponding to the original calibrations for each vapor via EDPCR. The identity of the vapor assigned to this synthetic response vector was determined by the shortest Euclidean distance. This procedure was performed iteratively (i.e. 1000 samples) to yield a statistical estimate of recognition rate (RR) for each array at each level of $\varepsilon$. Details of this methodology as applied to sensor-array evaluations can be found elsewhere $[25,26,31,32]$.

For the (virtual) dual NW-CR array, the ratio of normalized responses at the two different temperatures was treated as a single response variable. However, error was superimposed on each response separately, as above, to produce the error-enhanced ratio. Since the NW-CR response ratio is independent of concentration (see below), it was not necessary to vary the concentration when generating the population of error enhanced responses. Assigning a vapor identity to the synthetic NW-CR array responses was based merely on how close the value of the synthetic ratio was to each of the true (i.e. calibrated) ratios.

For assigning vapor identities to synthetic responses generated from a hybrid array, the sum of the normalized distances between the error-enhanced synthetic response vector and each of the corresponding calibrated response vectors for the sensors under consideration (i.e. the response ratio for the NW-CR array and the principal component for the MPN-CR array) was calculated. As above, the synthetic response was assigned to the vapor for which this sum was the smallest.

\section{Results and discussion}

The baseline conductance varied significantly among the tested NW-CR devices, ranging from 0.005 to $1 \mu \mathrm{S}$ at $215^{\circ} \mathrm{C}$. This variation undoubtedly arises from deviceto-device variations in the number of NWs involved in 


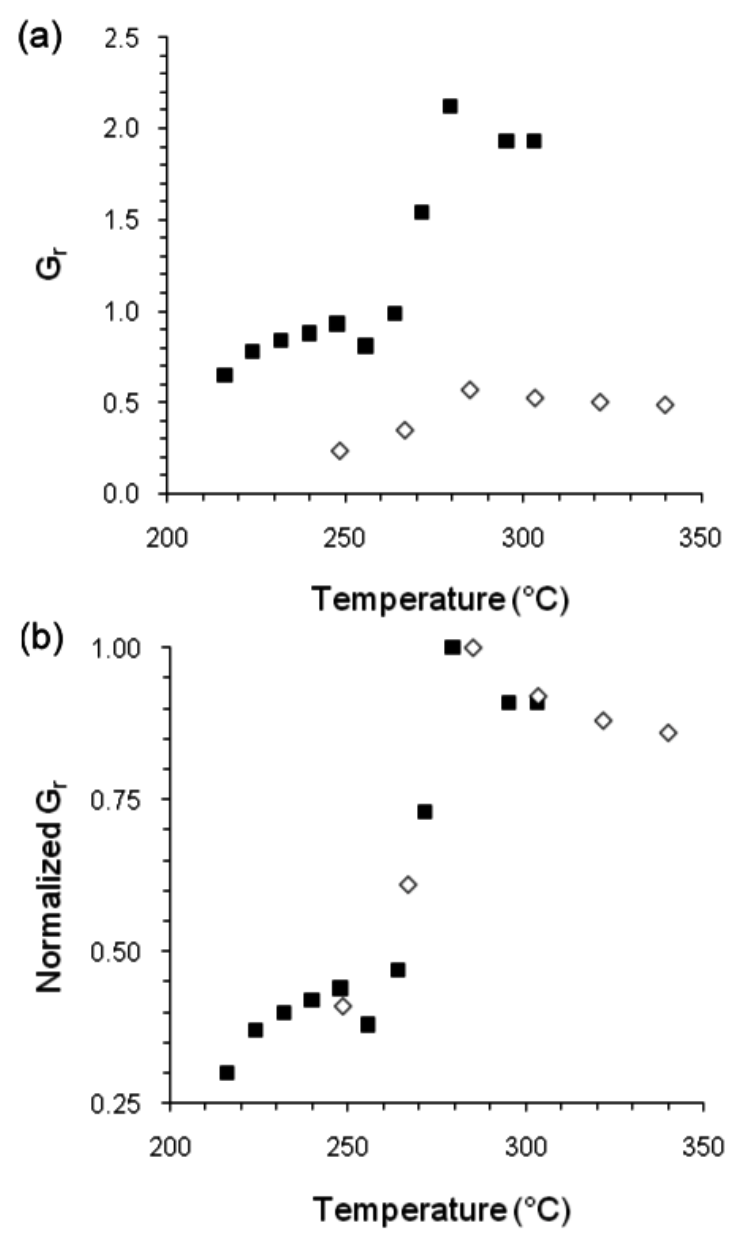

Figure 2. (a) Raw and (b) normalized relative responses $\left(G_{\mathrm{r}}=\Delta G / G\right)$ to nitromethane as a function of temperature for two NW-CR devices (filled squares, device 1, 240 ppm; unfilled diamonds, device 2, $360 \mathrm{ppm}$ ). For (b), the responses shown in (a) were normalized to the response at $280^{\circ} \mathrm{C}$ for device 1 and $285^{\circ} \mathrm{C}$ for device 2 .

charge conduction between the $\mathrm{CR}$ electrodes. The observed temperature dependence of baseline conductance is similar to that in previous reports [33], with conductance increasing sharply with increasing temperature (data not shown).

Initial testing characterized the temperature dependence of the NW-CR vapor sensitivity to nitromethane. Two NW CRs were exposed to nitromethane vapors at discrete operating temperatures ranging from 170 to $360{ }^{\circ} \mathrm{C}$. The exposure concentration was $240 \mathrm{ppm}$ for the first device and was inadvertently increased to $360 \mathrm{ppm}$ for the other. NW-CR responses achieved steady state within $\sim 20$ s of the initial response at all temperatures. Recoveries required up to $5 \mathrm{~min}$ to reach the pre-exposure baseline, which is consistent with other reports $[3,5]$. The sensitivity exhibits a nonlinear temperature dependence and passes through a maximum at $\sim 280^{\circ} \mathrm{C}$ for both devices (figure 2(a)). Although the raw sensitivities differ significantly between the two devices, by normalizing the response at any temperature to that at the temperature of maximum sensitivity (i.e. $280^{\circ} \mathrm{C}$ for device A and $285^{\circ} \mathrm{C}$ for device $\mathrm{B}$ ), the temperature dependent sensitivities of the two devices are nearly superimposable,
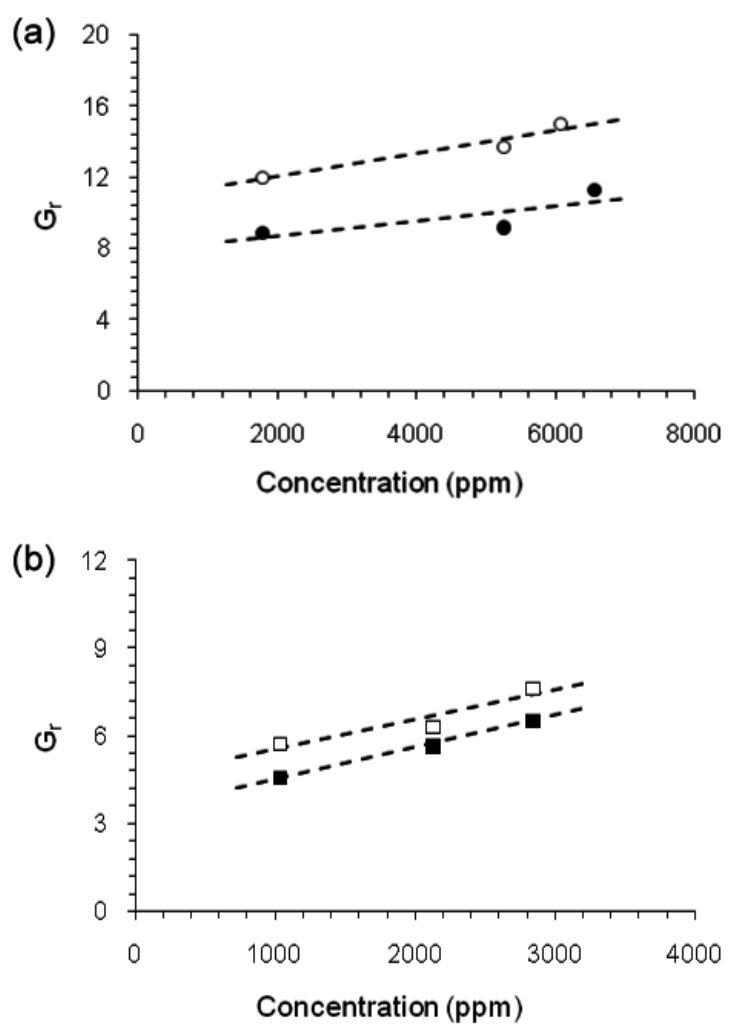

Figure 3. NW-CR calibration curves for (a) n-hexane and (b) toluene at $215^{\circ} \mathrm{C}$ (filled symbols) and $280^{\circ} \mathrm{C}$ (unfilled symbols). Lines represent the least-squares fit of the data $\left(r^{2}>0.92\right.$ in all cases). Response ratios do not vary significantly with concentration.

as shown in figure 2(b). This is not surprising, because the deposition process creates a stochastic distribution of nanowires (size and number) carrying current in each bridge. By the same token, the ratio of responses of a given device at two different temperatures is a useful metric for comparing vapor responses across devices, because it corrects for inherent differences in the mobilities and number of charge carriers in each device. Therefore, all subsequent NW-CR vapor exposures were performed at the temperatures at which the lowest and highest responses to nitromethane were observed, i.e. 215 and $280^{\circ} \mathrm{C}$.

Figures 3(a) and (b) show the NW-CR responses to n-hexane and toluene, respectively, over a range of concentrations at 215 and $280^{\circ} \mathrm{C}$. Although the response is greater at the higher temperature for any given concentration, the ratio of responses at the two different operating temperatures for a given vapor is nearly constant over the entire range of concentrations. Notably, however, the ratio differs between the two vapors: for toluene, the ratio of $G_{\mathrm{r}}$ values is $0.85 \pm 0.05$ and that for n-hexane is $0.72 \pm 0.04$. For nitromethane, a $G_{\mathrm{r}}$ ratio of 0.31 was obtained at $240 \mathrm{ppm}$ from the data presented in figure 2(a) for the same two temperatures. Thus, it is possible to differentiate the vapors on the basis of the response ratio over the concentration range tested.

Note that while the signal-to-noise ratios $\left(G_{\mathrm{r}}\right)$ are quite high, the slope sensitivities are relatively low over the concentration ranges measured for both of these vapors 

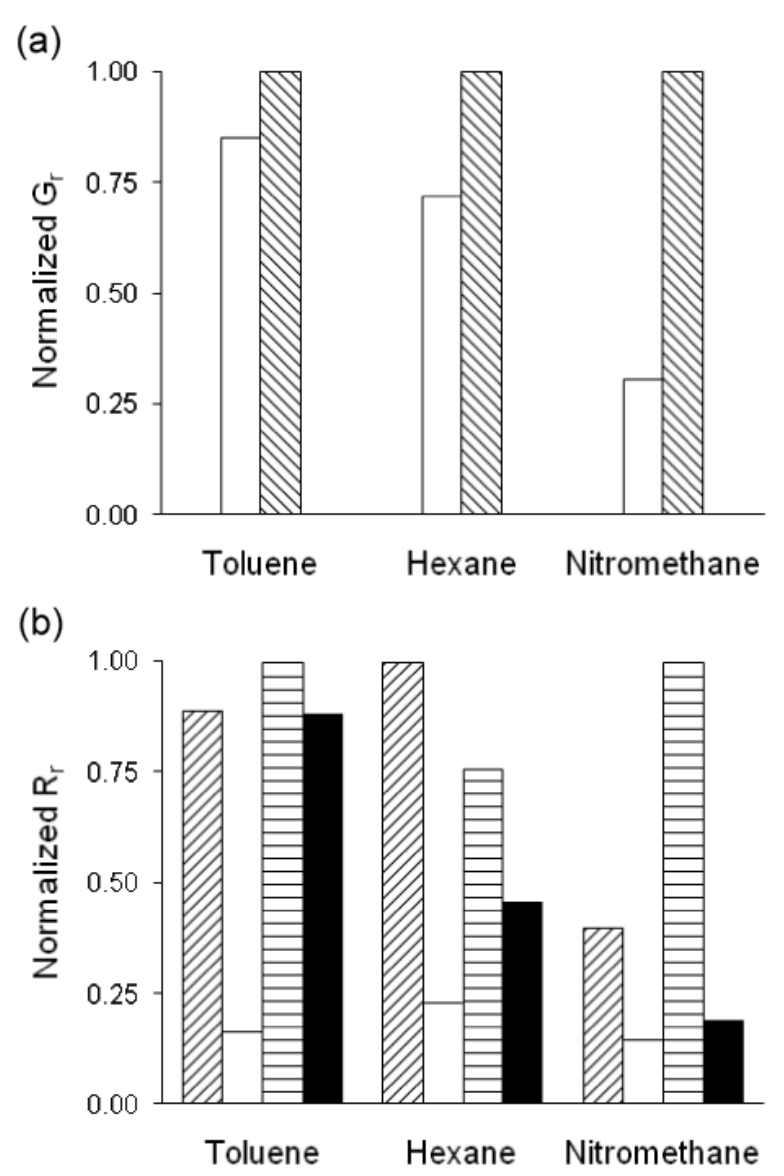

Figure 4. (a) Normalized relative response ratios for NW CRs exposed to the indicated vapors at $215^{\circ} \mathrm{C}$ (unfilled) and $280^{\circ} \mathrm{C}$ (cross-hatched); (b) normalized MPN-CR array sensitivities to the indicated vapors (in order, from left to right, $\mathrm{C} 8$, DPA, OPH, and HME).

at both temperatures, and the concentration resolution at any given temperature(s) is relatively low. It is apparent from the plots that these curves will become nonlinear at lower concentrations, with corresponding increases in slope sensitivity (i.e. resolution). But the ratios of responses at the different temperatures also may change. Thus, there could be a limit to the concentration range over which discrimination on the basis of temperature-dependent response ratios is possible (this range was not determined in this study).

Responses from the array of MPN CRs to the same three vapors were rapid and reversible, and varied linearly with concentration (linear regression $r^{2}$ values with forced zero $y$ intercepts are all $>0.97$ ), consistent with previous reports [16]. Response and recovery times were $\leqslant 20 \mathrm{~s}$ in all cases. The sensitivities derived from the responses of the two CRs coated with a given MPN differed by $<10 \%$ and were averaged for subsequent analyses. For n-hexane and toluene, MPNCR and NW-CR measurements were collected simultaneously. Sensors were exposed twice at each concentration to gather temperature-dependent NW-CR data. Since the duplicate MPN-CR sensor responses differed by $<7 \%$, the average values were used.

Figure 4 presents a series of bar charts that summarize the relevant response data. For the NW-CR array, the
Table 1. Average recognition rates (RR) with arrays of MPN CRs and NW CRs of different size (number) and composition for the discrimination of nitromethane, $\mathrm{n}$-hexane, and toluene as determined by Monte Carlo/EDPCR analysis ( $n=1000$ iterations) with different levels of superimposed random response error $(\varepsilon)$.

\begin{tabular}{|c|c|c|c|c|c|c|c|}
\hline & \multirow{2}{*}{\multicolumn{4}{|c|}{ Array size and composition }} & \multicolumn{3}{|c|}{ Recognition rate, $\varepsilon(\%)$} \\
\hline & & & & & 5 & 10 & 15 \\
\hline \multicolumn{8}{|c|}{2 sensors } \\
\hline - & DPA & HME & - & - & 99.9 & 95.0 & 88.0 \\
\hline - & - & HME & OPH & - & 99.8 & 93.3 & 87.2 \\
\hline C8 & - & - & OPH & - & 99.8 & 94.3 & 85.3 \\
\hline - & - & - & - & NW & 95.4 & 80.6 & 69.3 \\
\hline C8 & DPA & - & - & - & 91.3 & 80.7 & 74.4 \\
\hline - & DPA & - & OPH & - & 85.7 & 76.5 & 68.2 \\
\hline C8 & - & HME & - & - & 77.0 & 70.8 & 66.8 \\
\hline \multicolumn{8}{|c|}{3 sensors } \\
\hline C8 & - & HME & $\mathrm{OPH}$ & - & 100.0 & 99.5 & 97.2 \\
\hline - & DPA & HME & OPH & - & 100.0 & 98.6 & 93.9 \\
\hline C8 & DPA & - & $\mathrm{OPH}$ & - & 99.8 & 94.9 & 86.9 \\
\hline $\mathrm{C} 8$ & DPA & HME & - & 一 & 100.0 & 95.9 & 82.9 \\
\hline \multicolumn{8}{|c|}{4 sensors } \\
\hline C8 & DPA & HME & OPH & & 100.0 & 99.8 & 97.4 \\
\hline - & DPA & HME & - & NW & 100.0 & 98.6 & 95.5 \\
\hline $\mathrm{C} 8$ & - & HME & - & NW & 100.0 & 99.1 & 94.5 \\
\hline C8 & - & - & $\mathrm{OPH}$ & NW & 99.4 & 93.4 & 85.5 \\
\hline - & - & HME & $\mathrm{OPH}$ & NW & 99.0 & 91.3 & 84.7 \\
\hline - & DPA & - & $\mathrm{OPH}$ & NW & 98.6 & 89.8 & 81.3 \\
\hline C8 & DPA & - & - & NW & 94.6 & 83.1 & 77.3 \\
\hline \multicolumn{8}{|c|}{5 sensors } \\
\hline C8 & DPA & HME & - & NW & 100.0 & 98.9 & 95.4 \\
\hline $\mathrm{C} 8$ & - & HME & OPH & NW & 100.0 & 98.2 & 93.4 \\
\hline - & DPA & HME & OPH & NW & 99.7 & 95.3 & 90.0 \\
\hline C8 & DPA & - & OPH & NW & 99.4 & 94.8 & 87.8 \\
\hline \multicolumn{8}{|c|}{6 sensors } \\
\hline C8 & DPA & HME & OPH & NW & 100.0 & 98.0 & 94.4 \\
\hline
\end{tabular}

bars represent the relative sensitivities $\left(G_{\mathrm{r}}\right)$ at each test temperature for each vapor, normalized to the high-temperature $G_{\mathrm{r}}$ value. For the MPN-CR array the bars represent the relative sensitivities $\left(R_{\mathrm{r}} / \mathrm{ppm}\right)$ normalized to the sensor with the highest sensitivity for each vapor.

In order to compare the performance of different arrays properly, the number of sensors in each array must be the same. Since we are using the ratio of two NW CRs, in order to compare the vapor discrimination capabilities of this NW-CR array to those of the MPN CRs, it is necessary to restrict the number of MPN CRs in any array considered to two. Among the set of four MPN CRs, there are six possible dual-MPN-CR arrays, all of which were considered in succession.

Results of the Monte Carlo/EDPCR analyses, shown in table 1, reveal that the NW-CR array ranks fourth among the seven possible dual-sensor arrays in terms of RR. For $\varepsilon=5 \%$, the RR for the NW-CR array is $91.5 \%$, compared to an RR of 99.9\% for the dual MPN-CR array that used coatings of DPA and HME (best performance) and an RR of only $75.9 \%$ for the MPN-CR array that used coatings of C8 and HME (worst performance). The confusion matrix for the NW-CR array (not shown) indicates that it can distinguish nitromethane from the other two vapors without error (i.e. RR $=100 \%$ ) but confuses n-hexane and toluene at a high rate (i.e. the $R R$ values were 
both $\sim 88 \%$ ). A similar pattern of confusion is exhibited by the lower-ranked dual MPN-CR arrays. As the value of $\varepsilon$ increases the performance of all arrays declines, and at $\varepsilon=15 \%$ even the highest-ranked MPN-CR array exhibits unacceptably low RR values (i.e. $<90 \%$ ).

Analyses were then performed of arrays with larger numbers of sensors, including hybrid arrays consisting of 46 sensors (note: the smallest hybrid array considered was that consisting of two MPN CRs and the two NW CRs). The results are summarized in table 1 . All of the 3- and 4-sensor arrays are shown to perform quite well at the lowest level of superimposed error. As $\varepsilon$ increases, however, differences in performance emerge. The two highest ranked 4-sensor arrays are the 'all-MPN' array, with coatings of DPA, HME, C8, and $\mathrm{OPH}$, and the hybrid array that combines the DPA-and HME-coated CRs with the NW CR. Both give RR values $>95 \%$, even for $\varepsilon=15 \%$, and their performance is not statistically significantly different (i.e. the $95 \%$ confidence intervals around the average RR values overlap for all values of $\varepsilon)$. The performance of the third-highest ranked 4-sensor array (i.e. DPA, HME, and NW) is not significantly different from that of the higher ranking arrays, but its average RR value falls off at the highest $\varepsilon$ level, indicating somewhat less diversity. It is also noteworthy that the two highest-ranked 3-sensor MPNCR arrays perform as well as the three highest-ranked 4-sensor arrays. On the other hand, the three lowest-ranked 4-sensor arrays show greater sensitivity to the level of superimposed error, and their performance declines significantly for $\varepsilon>5 \%$.

Thus, several arrays could be used for this vapor discrimination problem as long as there is only a small amount of variation in sensor responses from sample to sample (i.e. a small value of $\varepsilon$ ). The highest ranked arrays are more resilient to such variations. Most importantly, the best 4-sensor hybrid array performs no better (or worse) than the best 3- or 4-sensor array consisting entirely of MPN CRs.

Interestingly, there appears to be no benefit to using more than three sensors in terms of recognition or resiliency to response error. Although this is largely due to the relatively simple problem being posed, which does not require additional input for its resolution, it illustrates the diminishing yield in diversity with increasing array size. Similar findings have been reported in attempting to discriminate among larger sets of individual vapors and their simple mixtures with other single- and multi-transducer arrays employing sorptive interface layers [25, 26, 31, 32].

\section{Conclusions}

Contact-printed tin-oxide NW-CR vapor sensors on membrane substrates with integrated heaters were fabricated, and the temperature dependence of the baseline conductance and sensitivity to each of three different organic vapors were characterized. The vapor sensitivity differed significantly among replicate NW-CR devices, due to differences in the number, size, and orientation of NWs involved in transporting charge across the electrode gap. However, taking the ratio of responses at two different temperatures yielded deviceindependent sensitivities that differed among the three test vapors examined.
The ability to discriminate among the test vapors on the basis of these response ratios was greater than that of some of the dual MPN-CR arrays to which it was compared, but less than others. Importantly, the best 4-sensor hybrid array one could create from a combination of NW and MPN CRs yielded vapor recognition rates that were not significantly different from those of the best 3- or 4-sensor array composed entirely of MPN CRs. Furthermore, the 6-sensor hybrid array showed no improvement in performance over the best 3- and 4-sensor arrays.

Thus, this preliminary study suggests that there is little or no advantage to combining tin-oxide NW CRs with MPN CRs in hybrid arrays for vapor recognition compared to using arrays consisting entirely of MPN CRs. The question of whether the performance of an NW-CR array would improve by use of devices operating at more than two temperatures or by rapid modulation of the temperature of a single device [8], remains unanswered and may be worth further investigation. Tests of mixtures, where the mixture components must be resolved from their composite, present a more difficult problem for any sensor array $[25,30]$, and would form the basis of more rigorous future assessments of performance.

\section{Acknowledgments}

The authors express their heartfelt appreciation to Hungwei Chang for his assistance in device testing, Jonathan Bryant-Genevier for his assistance in testing and data collection, and Katharine Beach and Sung-Jin Kim for their invaluable assistance in device fabrication. This work was supported through the Michigan Center for Wireless Integrated Microsystems by the Engineering Research Centers Program of the National Science Foundation under Award Number ERC-9986866. Additional support was provided by the Science and Technology Directorate, Department of Homeland Security under Cooperative Agreement 06-G024. Devices described in this report were fabricated in the Lurie Nanofabrication Facility, a member of the National Nanotechnology Infrastructure Network, which is supported by the National Science Foundation.

\section{References}

[1] Barsan N and Weimar U 2001 J. Electroceram. 7143

[2] Hernandez-Ramirez F, Prades J D, Jimenez-Diaz R, Fischer T, Romano-Rodriguez A, Mathur S and Morante J R 2009 Phys. Chem. 117105

[3] Meier D C, Semancik S, Button B, Strelcov E and Kolmakov A 2007 Appl. Phys. Lett. 91063118

[4] Sberveglieri G, Baratto C, Comini E, Faglia G, Ferroni M, Pardo M, Ponzoni A and Vomiero A 2009 Thin Solid Films $\mathbf{5 1 7} 6156$

[5] Kolmakov A, Zhang Y, Cheng G and Moskovits M 2003 Adv. Mater. 15997

[6] Ryu K, Zhang D and Zhou C 2008 Appl. Phys. Lett. 92093111

[7] Qin L, Xu J, Dong X, Pan Q, Cheng Z, Xiang Q and Li F 2008 Nanotechnology 19185705

[8] Benkstein K D, Raman B, Lahr D L, Bonevich J E and Semancik S 2009 Sensors Actuators B 13748

[9] Wohltjen H and Snow A W 1998 Anal. Chem. 702856 
[10] Joseph Y, Krasteva N, Besnard I, Guse B, Rosenberger M, Wild U, Knop-Gericke A, Schlogl R, Krustev R, Yasuda A and Vossmeyer T 2004 Faraday Discuss. 12577

[11] Han L, Shi X, Wu W, Kirk F, Luo J, Wang L, Mott D, Cousineau L, Lim S and Lu S 2005 Sensors Actuators B 106431

[12] Ibañez F J, Gowrishetty U, Crain M M, Walsh K M and Zamborini F P 2005 Anal. Chem. 78753

[13] Lu C-J, Steinecker W H, Tian W-C, Oborny M C, Nichols J M, Agah M, Potkay J A, Chan H K L, Driscoll J, Sacks R D, Wise K D, Pang S W and Zellers E T 2005 Lab Chip 51123

[14] Yang C, Li C and Lu C 2006 Anal. Chim. Acta 56517

[15] Han L, Daniel D R, Maye M M and Zhong C-J 2001 Anal. Chem. 734441

[16] Steinecker W H, Rowe M P and Zellers E T 2007 Anal. Chem. 794977

[17] Joseph Y, Peić A, Chen X, Michl J, Vossmeyer T and Yasuda A 2007 J. Phys. Chem. C 11112855

[18] Covington E, Bohrer F I, Xu C, Zellers E T and Kurdak C 2010 Lab Chip 10 3058-60

[19] Cai Q Y and Zellers E T 2002 Anal. Chem. 743533

[20] Schierbaum K D, Gerlach A, Haug M and Göpel W 1992 Sensors Actuators A 31130
[21] Haug M, Schierbaum K D, Gauglitz G and Göpel W 1993 Sensors Actuators B 11383

[22] Zhou R, Hierlemann A, Weimar U and Göpel W 1996 Sensors Actuators B 34356

[23] Ulmer H, Mitrovics J, Noetzel G, Weimar U and Göpel W 1997 Sensors Actuators B $\mathbf{4 3} 24$

[24] Pardo M, Kwong L, Sberveglieri G, Brubaker K, Schneider J, Penrose W and Stetter J 2005 Sensors Actuators B 106136

[25] Jin C, Kurzawski P, Hierlemann A and Zellers E T 2008 Anal. Chem. 80227

[26] Jin C and Zellers E T 2008 Anal. Chem. 807283

[27] Dattoli E N, Wan Q, Guo W, Chen Y, Pan X and Lu W 2007 Nano Lett. 72463

[28] Fan Z, Ho J C, Jacobson Z A, Yerushalmi R, Alley R L, Razavi H and Javey A 2008 Nano Lett. 820

[29] Zhong Q, Steinecker W H and Zellers E T 2009 Analyst 134283

[30] Rowe M P, Plass K E, Kim K, Kurdak C, Zellers E T and Matzger A J 2004 Chem. Mater. 163513

[31] Park J, Groves W A and Zellers E T 1999 Anal. Chem. 713877

[32] Hsieh M-D and Zellers E T 2004 Anal. Chem. 761885

[33] Wan Q, Dattoli E and Lu W 2008 Small 4451 